\title{
A Pfaffian-Hafnian Analogue of Borchardt's Identity
}

\section{Masao ISHIKAWA}

Faculty of Education, Tottori University

Koyama, Tottori, Japan

ishikawa@fed.tottori-u.ac.jp

\section{Hiroyuki KAWAMUKO}

Faculty of Education, Mie University

Tsu, Mie, Japan

kawam@edu.mie-u.ac.jp

\section{Soichi OKADA}

Graduate School of Mathematics, Nagoya University

Chikusa-ku, Nagoya, Japan

okada@math.nagoya-u.ac.jp

Submitted: Sep 13, 2004; Accepted: Jun 6, 2005 ; Published: Jun 14, 2005

Mathematics Subject Classifications: $05 \mathrm{E} 05$

\begin{abstract}
We prove

$$
\operatorname{Pf}\left(\frac{x_{i}-x_{j}}{\left(x_{i}+x_{j}\right)^{2}}\right)_{1 \leq i, j \leq 2 n}=\prod_{1 \leq i<j \leq 2 n} \frac{x_{i}-x_{j}}{x_{i}+x_{j}} \cdot \operatorname{Hf}\left(\frac{1}{x_{i}+x_{j}}\right)_{1 \leq i, j \leq 2 n}
$$

(and its variants) by using complex analysis. This identity can be regarded as a Pfaffian-Hafnian analogue of Borchardt's identity and as a generalization of Schur's identity.
\end{abstract}

\section{Introduction}

Determinant and Pfaffian identities play a key role in combinatorics and the representation theory (see, for example, [4], [5], [6], [8], [10], [11]). Among such determinant identities, the central ones are Cauchy's determinant identities ([2])

$$
\begin{aligned}
& \operatorname{det}\left(\frac{1}{x_{i}+y_{j}}\right)_{1 \leq i, j \leq n}=\frac{\prod_{1 \leq i<j \leq n}\left(x_{j}-x_{i}\right)\left(y_{j}-y_{i}\right)}{\prod_{i, j=1}^{n}\left(x_{i}+y_{j}\right)}, \\
& \operatorname{det}\left(\frac{1}{1-x_{i} y_{j}}\right)_{1 \leq i, j \leq n}=\frac{\prod_{1 \leq i<j \leq n}\left(x_{j}-x_{i}\right)\left(y_{j}-y_{i}\right)}{\prod_{i, j=1}^{n}\left(1-x_{i} y_{j}\right)} .
\end{aligned}
$$


C. W. Borchardt [1] gave a generalization of Cauchy's identities:

$$
\begin{gathered}
\operatorname{det}\left(\frac{1}{\left(x_{i}+y_{j}\right)^{2}}\right)_{1 \leq i, j \leq n}=\frac{\prod_{1 \leq i<j \leq n}\left(x_{j}-x_{i}\right)\left(y_{j}-y_{i}\right)}{\prod_{i, j=1}^{n}\left(x_{i}+y_{j}\right)} \cdot \operatorname{perm}\left(\frac{1}{x_{i}+y_{j}}\right)_{1 \leq i, j \leq n}, \\
\operatorname{det}\left(\frac{1}{\left(1-x_{i} y_{j}\right)^{2}}\right)_{1 \leq i, j \leq n}=\frac{\prod_{1 \leq i<j \leq n}\left(x_{j}-x_{i}\right)\left(y_{j}-y_{i}\right)}{\prod_{i, j=1}^{n}\left(1-x_{i} y_{j}\right)} \cdot \operatorname{perm}\left(\frac{1}{1-x_{i} y_{j}}\right)_{1 \leq i, j \leq n} .
\end{gathered}
$$

Here perm $A$ is the permanent of a square matrix $A$ defined by

$$
\operatorname{perm} A=\sum_{\sigma \in \mathcal{S}_{n}} a_{1 \sigma(1)} a_{2 \sigma(2)} \cdots a_{n \sigma(n)}
$$

This identity (3) is used when we evaluate the determinants appearing in the 0enumeration of alternating sign matrices (see [11]).

I. Schur [12] gave a Pfaffian analogue of Cauchy's identity (1) in his study of projective representations of the symmetric groups. Schur's Pfaffian identity and its variant $([9],[14])$ are

$$
\begin{gathered}
\operatorname{Pf}\left(\frac{x_{j}-x_{i}}{x_{j}+x_{i}}\right)_{1 \leq i, j \leq 2 n}=\prod_{1 \leq i<j \leq 2 n} \frac{x_{j}-x_{i}}{x_{j}+x_{i}}, \\
\operatorname{Pf}\left(\frac{x_{j}-x_{i}}{1-x_{i} x_{j}}\right)_{1 \leq i, j \leq 2 n}=\prod_{1 \leq i<j \leq 2 n} \frac{x_{j}-x_{i}}{1-x_{i} x_{j}} .
\end{gathered}
$$

In this note, we give identities which can be regarded as Pfaffian analogues of Borchardt's identities (3), (4) and as generalizations of Schur's identities (5), (6).

Theorem 1.1. Let $n$ be a positive integer. Then we have

$$
\begin{gathered}
\operatorname{Pf}\left(\frac{x_{i}-x_{j}}{\left(x_{i}+x_{j}\right)^{2}}\right)_{1 \leq i, j \leq 2 n}=\prod_{1 \leq i<j \leq 2 n} \frac{x_{i}-x_{j}}{x_{i}+x_{j}} \cdot \operatorname{Hf}\left(\frac{1}{x_{i}+x_{j}}\right)_{1 \leq i, j \leq 2 n}, \\
\operatorname{Pf}\left(\frac{x_{i}-x_{j}}{\left(1-x_{i} x_{j}\right)^{2}}\right)_{1 \leq i, j \leq 2 n}=\prod_{1 \leq i<j \leq 2 n} \frac{x_{i}-x_{j}}{1-x_{i} x_{j}} \cdot \operatorname{Hf}\left(\frac{1}{1-x_{i} x_{j}}\right)_{1 \leq i, j \leq 2 n} .
\end{gathered}
$$

Here Hf $A$ denotes the Hafnian of a symmetric matrix $A$ defined by

$$
\text { Hf } A=\sum_{\sigma \in \mathcal{F}_{2 n}} a_{\sigma(1) \sigma(2)} a_{\sigma(3) \sigma(4)} \cdots a_{\sigma(2 n-1) \sigma(2 n)},
$$

where $\mathcal{F}_{2 n}$ is the set of all permutations $\sigma$ satisfying $\sigma(1)<\sigma(3)<\cdots<\sigma(2 n-1)$ and $\sigma(2 i-1)<\sigma(2 i)$ for $1 \leq i \leq n$.

\section{Proof}

In this section, we prove the identity (7) in Theorem 1.1 by using complex analysis. The other identity (8) is shown by the same method, and also derived from more 
general identity (18) in Theorem 3.2, which follows from (7). So we omit the proof of (8) here.

Hereafter we put

$$
A=\left(\frac{x_{i}-x_{j}}{\left(x_{i}+x_{j}\right)^{2}}\right)_{1 \leq i, j \leq 2 n}, \quad B=\left(\frac{1}{x_{i}+x_{j}}\right)_{1 \leq i, j \leq 2 n} .
$$

For an $2 n \times 2 n$ symmetric (or skew-symmetric) matrix $M=\left(m_{i j}\right)$ and distinct indices $i_{1}, \cdots, i_{r}$, we denote by $M^{i_{1}, \cdots, i_{r}}$ the $(2 n-r) \times(2 n-r)$ matrix obtained by removing the rows and columns indexed by $i_{1}, \cdots, i_{r}$.

First we show two lemmas by using complex analysis. These lemmas hold in the rational function field $\mathbb{Q}\left(x_{1}, \ldots, x_{2 n}, z\right)$ and they may be shown in a purely algebraic way, but we found that complex analysis is very efficient for a compact proof.

\section{Lemma 2.1.}

$$
\sum_{\substack{1 \leq k, l \leq 2 n \\ k \neq l}} \frac{1}{\left(x_{k}-z\right)\left(x_{l}+z\right)} \operatorname{Hf}\left(B^{k, l}\right)=\operatorname{Hf}(B) \cdot \sum_{k=1}^{2 n} \frac{2 x_{k}}{x_{k}^{2}-z^{2}} .
$$

Proof. Let us denote by $F(z)$ (resp. $G(z)$ ) the left (resp. right) hand side of (9), and regard $F(z)$ and $G(z)$ as rational functions in the complex variable $z$, where $x_{1}, \cdots, x_{2 n}$ are distinct complex numbers. Then $F(z)$ and $G(z)$ have poles at $z= \pm x_{1}, \cdots, \pm x_{2 n}$ of order 1 . The residues of $F(z)$ at $z= \pm x_{m}$ are given by

$$
\begin{aligned}
& \operatorname{Res}_{z=x_{m}} F(z)=-\sum_{\substack{1 \leq l \leq 2 n \\
l \neq m}} \frac{1}{x_{l}+x_{m}} \operatorname{Hf}\left(B^{m, l}\right), \\
& \operatorname{Res}_{z=-x_{m}} F(z)=\sum_{\substack{1 \leq k \leq 2 n \\
k \neq m}} \frac{1}{x_{k}+x_{m}} \operatorname{Hf}\left(B^{k, m}\right) .
\end{aligned}
$$

By considering the expansion of $\operatorname{Hf}(B)$ along the $m$ th row/column, we have

$$
\operatorname{Res}_{z=x_{m}} F(z)=-\operatorname{Hf}(B), \quad \operatorname{Res}_{z=-x_{m}} F(z)=\operatorname{Hf}(B) .
$$

On the other hand, the residues of $G(z)$ at $z= \pm x_{m}$ are given by

$$
\begin{gathered}
\operatorname{Res}_{z=x_{m}} G(z)=-\operatorname{Hf}(B) \cdot \frac{2 x_{m}}{2 x_{m}}=-\operatorname{Hf}(B), \\
\operatorname{Res}_{z=-x_{m}} G(z)=\operatorname{Hf}(B) \cdot \frac{2 x_{m}}{2 x_{m}}=\operatorname{Hf}(B) .
\end{gathered}
$$

Since $\lim _{z \rightarrow \infty} F(z)=\lim _{z \rightarrow \infty} G(z)=0$, we conclude that $F(z)=G(z)$.

Lemma 2.2. If $n$ is a positive integer, then

$$
\sum_{k=1}^{2 n-1} \frac{x_{k}-z}{\left(x_{k}+z\right)^{2}} \prod_{\substack{1 \leq i \leq 2 n-1 \\ i \neq k}} \frac{x_{k}+x_{i}}{x_{k}-x_{i}} \cdot \operatorname{Hf}\left(B^{k, 2 n}\right)=\prod_{i=1}^{2 n-1} \frac{x_{i}-z}{x_{i}+z} \sum_{k=1}^{2 n-1} \frac{1}{x_{k}+z} \operatorname{Hf}\left(B^{k, 2 n}\right)
$$


Proof. Let $P(z)$ (resp. $Q(z)$ ) be the left (resp. right) hand side of (10), and regard $P(z)$ and $Q(z)$ as rational functions in $z$, where $x_{1}, \cdots, x_{2 n-1}$ are distinct complex numbers. Then $P(z)$ and $Q(z)$ have poles at $z=-x_{1}, \cdots,-x_{2 n-1}$ of order 2 . Thus, for a fixed $m$ such that $1 \leq m \leq 2 n-1$, we can write

$$
\begin{aligned}
& P(z)=\frac{p_{2}}{\left(z+x_{m}\right)^{2}}+\frac{p_{1}}{z+x_{m}}+O\left(z+x_{m}\right), \\
& Q(z)=\frac{q_{2}}{\left(z+x_{m}\right)^{2}}+\frac{q_{1}}{z+x_{m}}+O\left(z+x_{m}\right),
\end{aligned}
$$

in a neighborhood of $z=-x_{m}$. Now we compute the coefficients $p_{2}, p_{1}, q_{2}$ and $q_{1}$, and prove $p_{2}=q_{2}, p_{1}=q_{1}$.

By using the relation

$$
\frac{x_{m}-z}{\left(x_{m}+z\right)^{2}}=\frac{2 x_{m}}{\left(x_{m}+z\right)^{2}}-\frac{1}{x_{m}+z}
$$

we see that

$$
\begin{aligned}
& p_{2}=2 x_{m} \prod_{\substack{1 \leq i \leq 2 n-1 \\
i \neq m}} \frac{x_{m}+x_{i}}{x_{m}-x_{i}} \cdot \operatorname{Hf}\left(B^{m, 2 n}\right), \\
& p_{1}=-\prod_{\substack{1 \leq i \leq 2 n-1 \\
i \neq m}} \frac{x_{m}+x_{i}}{x_{m}-x_{i}} \cdot \operatorname{Hf}\left(B^{m, 2 n}\right) .
\end{aligned}
$$

Next we deal with

$$
Q(z)=\frac{x_{m}-z}{x_{m}+z} \times \prod_{\substack{1 \leq i \leq 2 n-1 \\ i \neq m}} \frac{x_{i}-z}{x_{i}+z} \times \sum_{k=1}^{2 n-1} \frac{1}{x_{k}+z} \operatorname{Hf}\left(B^{k, 2 n}\right) .
$$

The first factor can be written in the form

$$
\frac{x_{m}-z}{x_{m}+z}=\frac{2 x_{m}}{x_{m}+z}-1 \text {. }
$$

By using the Taylor expansion $\log (1-t)=-t+O\left(t^{2}\right)$, we have

$$
\begin{aligned}
& \log \frac{x_{i}-z}{x_{m}+x_{i}}=-\frac{z+x_{m}}{x_{i}+x_{m}}+O\left(\left(z+x_{m}\right)^{2}\right), \\
& \log \frac{x_{i}+z}{x_{m}-x_{i}}=\frac{z+x_{m}}{x_{i}-x_{m}}+O\left(\left(z+x_{m}\right)^{2}\right) .
\end{aligned}
$$

Hence we see that

$$
\log \left(\frac{x_{i}-z}{x_{i}+z} / \frac{x_{i}+x_{m}}{x_{i}-x_{m}}\right)=-\frac{2 x_{i}}{x_{i}^{2}-x_{m}^{2}}\left(z+x_{m}\right)+O\left(\left(z+x_{m}\right)^{2}\right) .
$$

Therefore the second factor of $Q(z)$ has the form

$$
\begin{aligned}
& \prod_{\substack{1 \leq i \leq 2 n-1 \\
i \neq m}} \frac{x_{i}-z}{x_{i}+z} \\
& \quad=\prod_{\substack{1 \leq i \leq 2 n-1 \\
i \neq m}} \frac{x_{i}+x_{m}}{x_{i}-x_{m}} \cdot\left\{1-\sum_{\substack{1 \leq k \leq 2 n-1 \\
k \neq m}} \frac{2 x_{k}}{x_{k}^{2}-x_{m}^{2}} \cdot\left(z+x_{m}\right)+O\left(\left(z+x_{m}\right)^{2}\right)\right\} .
\end{aligned}
$$


Since we have

$$
\frac{1}{x_{k}+z}=\frac{1}{x_{k}-x_{m}}+O\left(z+x_{m}\right)
$$

the last factor of $Q(z)$ has the following expansion:

$$
\sum_{k=1}^{2 n-1} \frac{1}{x_{k}+z} \operatorname{Hf}\left(B^{k, 2 n}\right)=\frac{1}{x_{m}+z} \operatorname{Hf}\left(B^{m, 2 n}\right)+\sum_{\substack{1 \leq k \leq 2 n-1 \\ k \neq m}} \frac{1}{x_{k}-x_{m}} \operatorname{Hf}\left(B^{k, 2 n}\right)+O\left(z+x_{m}\right) .
$$

Combining these expansions, we have

$$
q_{2}=2 x_{m} \prod_{\substack{1 \leq i \leq 2 n-1 \\ i \neq m}} \frac{x_{i}+x_{m}}{x_{i}-x_{m}} \cdot \operatorname{Hf}\left(B^{m, 2 n}\right)
$$

and

$$
\begin{aligned}
q_{1}= & \prod_{\substack{1 \leq i \leq 2 n-1 \\
i \neq m}} \frac{x_{i}+x_{m}}{x_{i}-x_{m}} \\
& \times\left\{2 x_{m} \sum_{\substack{1 \leq k \leq 2 n-1 \\
k \neq m}} \frac{\operatorname{Hf}\left(B^{k, 2 n}\right)}{x_{k}-x_{m}}-2 x_{m} \operatorname{Hf}\left(B^{m, 2 n}\right) \sum_{\substack{1 \leq k \leq 2 n-1 \\
k \neq m}} \frac{2 x_{k}}{x_{k}^{2}-x_{m}^{2}}-\operatorname{Hf}\left(B^{m, 2 n}\right)\right\} .
\end{aligned}
$$

It follows from (11) and (13) that $p_{2}=q_{2}$. From (12) and (14), in order to prove the equality $p_{1}=q_{1}$, it is enough to show that

$$
\sum_{\substack{1 \leq k \leq 2 n-1 \\ k \neq m}} \frac{1}{x_{k}-x_{m}} \operatorname{Hf}\left(B^{k, 2 n}\right)=\operatorname{Hf}\left(B^{m, 2 n}\right) \sum_{\substack{1 \leq k \leq 2 n-1 \\ k \neq m}} \frac{2 x_{k}}{x_{k}^{2}-x_{m}^{2}} .
$$

By permuting the variables $x_{1}, \cdots, x_{2 n-1}$, we may assume that $m=2 n-1$. Then, by expanding the Hafnian on the left hand side along the last row/column, it is enough to show that

$$
\sum_{k=1}^{2 n-2} \frac{1}{x_{k}-x_{2 n-1}} \sum_{\substack{1 \leq l \leq 2 n-2 \\ l \neq k}} \frac{1}{x_{l}+x_{2 n-1}} \operatorname{Hf}\left(B^{k, l, 2 n-1,2 n}\right)=\operatorname{Hf}\left(B^{2 n-1,2 n}\right) \sum_{k=1}^{2 n-2} \frac{2 x_{k}}{x_{k}^{2}-x_{2 n-1}^{2}} .
$$

This follows from Lemma 2.1 (with $2 n$ replaced by $2 n-2$ and $z$ replaced by $x_{2 n-1}$ ), and we complete the proof of Lemma 2.2.

Now we are in the position to prove the identity (7) in Theorem 1.1.

Proof of (7). We proceed by induction on $n$.

Expanding the Pfaffian along the last row/column and using the induction hypothesis, we see

$$
\begin{aligned}
\operatorname{Pf}(A) & =\sum_{k=1}^{2 n-1}(-1)^{k-1} \frac{x_{k}-x_{2 n}}{\left(x_{k}+x_{2 n}\right)^{2}} \operatorname{Pf}\left(A^{k, 2 n}\right) \\
& =\sum_{k=1}^{2 n-1}(-1)^{k-1} \frac{x_{k}-x_{2 n}}{\left(x_{k}+x_{2 n}\right)^{2}} \prod_{\substack{1 \leq i<j \leq 2 n-1 \\
i, j \neq k}} \frac{x_{i}-x_{j}}{x_{i}+x_{j}} \operatorname{Hf}\left(B^{k, 2 n}\right) .
\end{aligned}
$$


By using the relation

$$
\prod_{\substack{1 \leq i<j \leq 2 n-1 \\ i, j \neq k}} \frac{x_{i}-x_{j}}{x_{i}+x_{j}}=(-1)^{k-1} \prod_{1 \leq i<j \leq 2 n-1} \frac{x_{i}-x_{j}}{x_{i}+x_{j}} \cdot \prod_{\substack{1 \leq i \leq 2 n-1 \\ i \neq k}} \frac{x_{k}+x_{i}}{x_{k}-x_{i}}
$$

we have

$$
\operatorname{Pf}(A)=\prod_{1 \leq i<j \leq 2 n-1} \frac{x_{i}-x_{j}}{x_{i}+x_{j}} \sum_{k=1}^{2 n-1} \frac{x_{k}-x_{2 n}}{\left(x_{k}+x_{2 n}\right)^{2}} \prod_{\substack{1 \leq i \leq 2 n-1 \\ i \neq k}} \frac{x_{k}+x_{i}}{x_{k}-x_{i}} \cdot \operatorname{Hf}\left(B^{k, 2 n}\right) .
$$

On the other hand, by expanding the Hafnian along the last row/column, we have

$$
\prod_{1 \leq i<j \leq 2 n} \frac{x_{i}-x_{j}}{x_{i}+x_{j}} \cdot \operatorname{Hf}(B)=\prod_{1 \leq i<j \leq 2 n} \frac{x_{i}-x_{j}}{x_{i}+x_{j}} \sum_{k=1}^{2 n-1} \frac{1}{x_{k}+x_{2 n}} \cdot \operatorname{Hf}\left(B^{k, 2 n}\right) .
$$

So it is enough to show the following identity:

$$
\sum_{k=1}^{2 n-1} \frac{x_{k}-x_{2 n}}{\left(x_{k}+x_{2 n}\right)^{2}} \prod_{\substack{1 \leq i \leq 2 n-1 \\ i \neq k}} \frac{x_{k}+x_{i}}{x_{k}-x_{i}} \cdot \operatorname{Hf}\left(B^{k, 2 n}\right)=\prod_{i=1}^{2 n-1} \frac{x_{i}-x_{2 n}}{x_{i}+x_{2 n}} \sum_{k=1}^{2 n-1} \frac{1}{x_{k}+x_{2 n}} \cdot \operatorname{Hf}\left(B^{k, 2 n}\right) .
$$

This identity follows from Lemma 2.2 and the proof completes.

\section{Generalization}

The Cauchy's identities (1) and (2), and the Borchardt's identities (3) and (4) are respectively unified in the following form. (D. Knuth [7] considered this type of generalization.)

Theorem 3.1. Let $f(x, y)=a x y+b x+c y+d$ be a nonzero polynomial. Then we have

$$
\begin{gathered}
\operatorname{det}\left(\frac{1}{f\left(x_{i}, y_{j}\right)}\right)_{1 \leq i, j \leq n}=(-1)^{n(n-1)}(a d-b c)^{n(n-1) / 2} \frac{\prod_{1 \leq i<j \leq n}\left(x_{j}-x_{i}\right)\left(y_{j}-y_{i}\right)}{\prod_{1 \leq i, j \leq n} f\left(x_{i}, y_{j}\right)} \\
\operatorname{det}\left(\frac{1}{f\left(x_{i}, y_{j}\right)^{2}}\right)_{1 \leq i, j \leq n}=(-1)^{n(n-1)}(a d-b c)^{n(n-1) / 2} \frac{\prod_{1 \leq i<j \leq n}\left(x_{j}-x_{i}\right)\left(y_{j}-y_{i}\right)}{\prod_{1 \leq i, j \leq n} f\left(x_{i}, y_{j}\right)} \\
\times \operatorname{perm}\left(\frac{1}{f\left(x_{i}, y_{j}\right)}\right)_{1 \leq i, j \leq n} .
\end{gathered}
$$

Similarly we can generalize the Schur's identities (5) and (6), and our identities (7) and (8).

Theorem 3.2. Let $g(x, y)=a x y+b(x+y)+c$ be a nonzero polynomial. Then we have

$$
\begin{aligned}
& \operatorname{Pf}\left(\frac{x_{j}-x_{i}}{g\left(x_{i}, x_{j}\right)}\right)_{1 \leq i, j \leq 2 n}=\left(b^{2}-a c\right)^{n(n-1)} \prod_{1 \leq i<j \leq 2 n} \frac{x_{j}-x_{i}}{g\left(x_{i}, x_{j}\right)}, \\
& \operatorname{Pf}\left(\frac{x_{j}-x_{i}}{g\left(x_{i}, x_{j}\right)^{2}}\right)_{1 \leq i, j \leq 2 n}=\left(b^{2}-a c\right)^{n(n-1)} \prod_{1 \leq i<j \leq 2 n} \frac{x_{j}-x_{i}}{g\left(x_{i}, x_{j}\right)} \cdot \operatorname{Hf}\left(\frac{1}{g\left(x_{i}, x_{j}\right)}\right)_{1 \leq i, j \leq 2 n} .
\end{aligned}
$$


This generalization (17) is given in [7].

Proof. We derive (17) and (18) from (5) and (7) respectively.

First we consider the case where $b^{2}-a c \neq 0$. Suppose that $a \neq 0$. Then, by putting

$$
A=\frac{1}{2}, \quad B=\frac{1}{2 a}\left(b+\sqrt{b^{2}-a c}\right), \quad C=a, \quad D=b-\sqrt{b^{2}-a c},
$$

and substituting

$$
x_{i} \rightarrow \frac{A x_{i}+B}{C x_{i}+D} \quad(1 \leq i \leq 2 n)
$$

in (5) and (7), we obtain (17) and (18). Similarly we can show the case where $c \neq 0$.

If $b^{2}-a c=0$ and $a \neq 0$, then we have

$$
g\left(x_{i}, x_{j}\right)=a^{-1}\left(a x_{i}+b\right)\left(a x_{j}+b\right) .
$$

Hence we can evaluate the left hand sides of (17) and (18) by using

$$
\operatorname{Pf}\left(x_{j}-x_{i}\right)_{1 \leq i, j \leq 2 n}= \begin{cases}x_{2}-x_{1} & \text { if } n=1 \\ 0 & \text { if } n \geq 2\end{cases}
$$

and obtain the equalities in (17) and (18). Similarly we can show the case where $b^{2}-a c=0$ and $c \neq 0$.

From (15) and (16), we have

$$
\operatorname{det}\left(\frac{1}{f\left(x_{i}, y_{j}\right)^{2}}\right)_{1 \leq i, j \leq n}=\operatorname{det}\left(\frac{1}{f\left(x_{i}, y_{j}\right)}\right)_{1 \leq i, j \leq n} \cdot \operatorname{perm}\left(\frac{1}{f\left(x_{i}, y_{j}\right)}\right)_{1 \leq i, j \leq n} .
$$

Since the matrix $\left(f\left(x_{i}, y_{j}\right)\right)_{1 \leq i, j \leq n}$ has rank at most 2 , this identity is the special case of the following theorem.

Theorem 3.3. (Carlitz and Levine [3]) Let $A=\left(a_{i j}\right)$ be a matrix of rank at most 2. If $a_{i j} \neq 0$ for all $i$ and $j$, we have

$$
\operatorname{det}\left(\frac{1}{a_{i j}^{2}}\right)_{1 \leq i, j \leq n}=\operatorname{det}\left(\frac{1}{a_{i j}}\right)_{1 \leq i, j \leq n} \cdot \operatorname{perm}\left(\frac{1}{a_{i j}}\right)_{1 \leq i, j \leq n} .
$$

From (17) and (18), we have

$$
\operatorname{Pf}\left(\frac{x_{j}-x_{i}}{g\left(x_{i}, x_{j}\right)^{2}}\right)_{1 \leq i, j \leq 2 n}=\operatorname{Pf}\left(\frac{x_{j}-x_{i}}{g\left(x_{i}, x_{j}\right)}\right)_{1 \leq i, j \leq 2 n} \cdot \operatorname{Hf}\left(\frac{1}{g\left(x_{i}, x_{j}\right)}\right)_{1 \leq i, j \leq 2 n} .
$$

It is a natural problem to find a Pfaffian-Hafnian analogue of Theorem 3.3. Also it is interesting to find more examples of a skew-symmetric matrix $X$ and a symmetric matrix $Y$ satisfying

$$
\operatorname{Pf}\left(x_{i j} y_{i j}\right)_{1 \leq i, j \leq 2 n}=\operatorname{Pf}\left(x_{i j}\right)_{1 \leq i, j \leq 2 n} \cdot \operatorname{Hf}\left(y_{i j}\right)_{1 \leq i, j \leq 2 n} .
$$

Recently there appeared a bijective proof of Borchardt's identity (see [13]). It will be an interesting problem to give a bijective proof of (7) and (8). 


\section{References}

[1] C. W. Borchardt, Bestimmung der symmetrischen Verbindungen vermittelst ihrer erzeugenden Funktion, J. Reine Angew. Math. 53 (1855), 193-198.

[2] A. L. Cauchy, Mémoire sur les fonctions altertées et sur les sommes alternées, Exercices Anal. et Phys. Math. 2 (1841), 151-159.

[3] L. Carlitz and J. Levine, An identity of Cayley, Amer. Math. Monthly 67 (1960), 571-573.

[4] M. Ishikawa, S. Okada and M. Wakayama, Applications of minor summation formulas I : Littlewood's formulas, J. Algebra 183 (1996), 193-216.

[5] M. Ishikawa and M. Wakayama, Applications of minor summation formulas II : Pfaffians and Schur polynomials, J. Combin. Theory Ser. A 88 (1999), 136-157.

[6] M. Ishikawa and M. Wakayama, Applications of minor summation formulas III : Plücker relations, lattice paths and Pfaffian identities, arXiv:math. C0/0312358, to appear in J. Combin. Theory Ser. A.

[7] D. Knuth, Overlapping Pfaffians, Electron. J. Combin. 3 (2) ("The Foata Festschrift") (1996), 151-163.

[8] C. Krattenthaler, Advanced determinant calculus, Sem. Lothar. Combin. 42 ("The Andrews Festschrift") (1999), Article B42q.

[9] D. Laksov, A. Lascoux and A. Thorup, On Giambelli's theorem on complete correlations, Acta Math. 162 (1989), 143-199.

[10] S. Okada, Application of minor summation formulas to rectangular-shaped representations of classical groups, J. Algebra 205 (1998), 337-367.

[11] S. Okada, Enumeration of symmetry classes of alternating sign matrices and characters of classical groups, arXiv:math.C0/0408234, to appear.

[12] I. Schur, Über die Darstellung der symmetrischen und der alternirenden Gruppe durch gebrochene lineare Substitutuionen, J. Reine Angew. Math. 139 (1911), 155-250.

[13] D. Singer, A bijective proof of Borchardt's identity, Electron. J. Combin. 11 (1), R48

[14] J. R. Stembridge, Non-intersecting paths, Pfaffians and plane partitions, Adv. Math. 83 (1990), 96-131. 